\title{
Air Traffic Controller Performance and Acceptability of Multiple UAS in a Simulated NAS Environment
}

\author{
Kim-Phuong L. Vu, Thomas Strybel, Dan \\ Chiappe, and Greg Morales \\ California State University Long Beach \\ 1250 N Bellflower Blvd \\ Long Beach, CA 90840 USA \\ +15629855021 \\ Kim.Vu@csulb.edu
}

\author{
Vernol Battiste ${ }^{12}$ and Robert Jay Shively ${ }^{1}$ \\ ${ }^{1}$ NASA Ames Research Center \\ ${ }^{2}$ San Jose State University Foundation \\ Moffet Field, CA USA \\ $+16056043666$ \\ Vernol.battiste@nasa.gov
}

\begin{abstract}
Previously, we showed that air traffic controllers (ATCos) rated UAS pilot verbal response latencies as acceptable when a $1.5 \mathrm{~s}$ delay was added to the UAS pilot responses, but a $5 \mathrm{~s}$ delay was rated as mostly unacceptable. In the present study we determined whether a $1.5 \mathrm{~s}$ added delay in the UAS pilots' verbal communications would affect ATCos interactions with UAS and other conventional aircraft when the number and speed of the UAS were manipulated. Eight radar-certified ATCos participated in this simulation. The ATCos managed a medium altitude sector containing arrival aircraft, en route aircraft, and one to four UAS. The UAS were conducting a surveillance mission and flew at either a "slow" or "fast" speed. We measured both UAS and conventional pilots' verbal communication latencies, and obtained ATCos' acceptability ratings for these latencies. Although the UAS pilot response latencies were longer than those of conventional pilots, the ATCos rated UAS pilot verbal communication latencies to be as acceptable as those of conventional pilots. Because the overall traffic load within the sector was held constant, ATCos only performed slightly worse when multiple UAS were in their sector compared to when only one UAS was in the sector. Implications of these findings for UAS integration in the NAS are discussed.
\end{abstract}

\section{Keywords}

Unmanned Aerial Systems; measured response, human-inthe-loop simulation

\section{INTRODUCTION}

Unmanned Aerial Systems (UAS) have a variety of practical uses such as land surveying, disaster response

\footnotetext{
Permission to make digital or hard copies of all or part of this work for personal or classroom use is granted without fee provided that copies are not made or distributed for profit or commercial advantage and that copies bear this notice and the full citation on the first page. To copy otherwise, or republish, to post on servers or to redistribute to lists, requires prior specific permission and/or a fee.

HCI-Aero 2014, date, city, state, USA.

Copyright 2014 ACM doi...\$5.00
}

assistance, law enforcement, and climate research. For example, in 2006 NASA's Ikhana UAS was employed to assist fire-fighting efforts by providing timely information on the progression of wildfires and their hot spots [1]. The recognized value of UAS has led to the passage of the FAA (Federal Aviation Administration) Modernization and Reform Act of 2012, which calls for standards for UAS certification and integration in the NAS (National Airspace System).

Currently, UAS may obtain approval from the FAA to operate within the NAS by either being granted an experimental airworthiness certificate for private sector operations, or a Certificate of Authorization (COA) for public sector operations. Neither of these methods, though, allows for UAS to have routine, unrestricted access to the NAS. For UAS to be integrated into the NAS, the "UAS will be required to act and respond as manned aircraft do" (p. 5) [2]. This requirement involves not only assessing the performance capabilities of the UAS, but also its communication characteristics when interacting with air traffic controllers (ATCos) [3].

In terms of communication with ATCos, although FAA regulations require pilots of conventional aircraft to respond promptly, they do not specify a precise time limit on communication delays or pilot execution of ATCo commands. If these guidelines are transferred to UAS operations, prompt responding needs to be further specified because UAS differ from manned aircraft in many respects [4], and these differences can have an impact on the timeliness of the UAS responses. For example, UAS pilots are not co-located with their aircraft and are required to interact with their aircraft using ground control stations that currently have complex control interfaces [1]. They lack many sensory cues and can only rely on their instrument indicators and cameras with limited spatial resolution and small fields of view. As a result, UAS pilots have less information about their aircraft's surrounding environment [5]. Moreover, UAS communication time can be affected by systemic aspects, such as whether the UAS is within the line of sight or communicating via satellite. Thus, UAS pilots may take longer than conventional pilots to 
determine whether they can safely carry out a command issued by an ATCo.

For conventional aircraft, pilot delays in verbal responding have been shown to negatively affect the performance of ATCos [6]. As a result, ATCos may adjust their communication style in response to pilot verbal delays by, for example, increasing the complexity of commands. However, this can result in more communication errors [7]. For UAS, two studies have examined the effect of simulated UAS pilot delays on ATCo acceptability ratings of their response time [3], as well as the impact of additional delays on ATCo performance [8].

For one, Shivley et al. [3] examined the pilot-ATCo interaction in terms of a "measured response" (MR). The MR reflects several time components from the end of the ATCo's command transmission to the first noticeable display of the aircraft executing the instruction on the ATCo's radar scope. The first MR component (MR1) is measured from the end of the controller's instruction to the beginning of the pilot's verbal response. MR2 is from the end of the controller's instruction to when the pilot begins a control input on the ground control station. MR3 is the interval between the end of the pilot's control input to the onset of the aircraft's execution of the maneuver. MR4 is the time from the initiation of the aircraft's execution of the maneuver to the maneuver appearing on the ATCo radar scope (see Figure 1). As shown in Figure 1, these MR components are affected by many factors. For the purpose of the present study we focus only on the first component, MR 1: Pilot verbal communication latency.

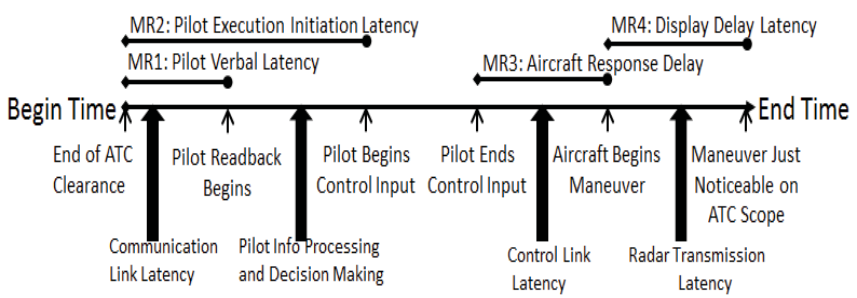

Figure 1. End-to-end response time for completion of ATC clearances. Figure based on Shively et al. (2013).

Shively et al. [3] had ATCos issue common commands to IFR-rated pilots who were trained to fly the UAS on a MUSIM (Multiple UAS simulator [9]) ground control station. The commands issued were route amendments, traffic calls, altitude changes, and radio frequency changes. The UAS pilots' verbal delay in responding to controllers averaged 2.5 seconds, a response time that was rated acceptable by ATCos. However, the acceptability ratings obtained in this simulation are not likely to reflect UAS operations in the NAS because both the pilots and ATCos were only issuing and responding to individual clearances in serial order. That is, the ATCos were not managing any other traffic, and the pilots were not performing any other tasks. ATCo acceptability of the MR1 component will likely differ in the presence of other traffic and with the addition of UAS communication delays.

$\mathrm{Vu}$ et al. [8] examined how short $(1.5 \mathrm{sec})$ and long $(5 \mathrm{sec})$ delays added to the pilot verbal latencies (MR1) and execution initiation latencies (MR2) influenced ATCo performance and their acceptability ratings of the UAS pilots relative to conventional pilots in a simulated NAS environment. They found that the ATCos rated UAS pilot verbal communication latencies to be more acceptable when the inserted delays were short $(1.5 \mathrm{~s})$ rather than long (5 s); however, the acceptability ratings were based on other features of the sectors being managed. For example, the ATCos rated the acceptability of UAS delays within a scenario based on all aircraft rather than the UAS alone, because differences between the acceptability ratings of UAS compared with conventional aircraft, were minimal, even though delays were added only to the UAS responses. Moreover, the ATCo acceptability ratings were correlated with measures of their performance in a scenario, for example, the number of losses of separation (LOS), number of step-ons that occurred, and the efficiency of their traffic management in the scenario was worse with long UAS delays.

The present study extends the work of Shively et al. [3] and $\mathrm{Vu}$ et al. [8] by examining the impact of multiple UAS in a simulated NAS environment. Only a short delay of $1.5 \mathrm{~s}$ was added to UAS pilot verbal communications since this value was found to be acceptable to ATCos. We manipulated both the number of UAS in the sector, (one, two or four), and their speeds. UAS flew at either "slow" speeds of $120 \mathrm{kts}$ (representing the characteristics of a Predator) or "fast" speeds of $240 \mathrm{kts}$ (representing the characteristics of a Global Hawk). We examined ATCo performance and acceptability ratings of UAS pilots' and conventional pilots' verbal response latencies (MR1) as a function of the number of UAS and the UAS speed in the sector. The data reported in this paper reflects only part of the data collected from the larger simulation.

\section{METHOD \\ Participants}

Eight radar-certified ATCos volunteered to participate in this simulation. The ATCos averaged over 25 years of military and civilian experience in air traffic management. All had prior experience with ZLA airspace during their active air traffic management period. The simulation lasted two days and participants were compensated $\$ 60$ per hour for their time.

\section{Design}

This simulation followed a 3 (Number of UAS: 1, 2, or 4) $\mathrm{x}$ 3 (Speed: Slow, Mixed, or Fast) within-subjects, repeated measure design (see Figure 2). ${ }^{1}$

\footnotetext{
1 As noted earlier in the paper, only a portion of the data from the full simulation is being reported here.
} 


\begin{tabular}{|c|c|c|c|}
\hline & \multicolumn{3}{|c|}{ Number of "Fast" UAS } \\
\hline No. UAS & None & Half & All \\
\hline $\mathbf{1}$ & 1 slow UAS & $\begin{array}{c}\text { 1 UAS (speed change } \\
\text { during scenario) }\end{array}$ & 1 fast UAS \\
\hline $\mathbf{2}$ & 2 slow UAS & $\begin{array}{c}\text { 1 slow UAS } \\
\text { 1 fast UAS }\end{array}$ & 2 fast UAS \\
\hline $\mathbf{4}$ & 4 slow UAS & $\begin{array}{c}\text { 2 slow UAS } \\
\text { 2 fast UAS }\end{array}$ & 4 fast UAS \\
\hline
\end{tabular}

Figure 2. Illustration of the 3 (No. of UAS) x 3 (Speed) design

\section{Simulation Environment}

The simulation was run in three separate rooms: one room to manage the simulation and run the ATCo participants, a second room for conventional pilots, and the third for UAS pilots. All pilots were trained experimental confederates.

The simulation was run using the Multi Aircraft Control System (MACS [10]), creating a medium-fidelity environment. MACS simulated a controllers DSR display for sector ZLA 20 (Figure 3). The multi-aircraft mode was used by pseudopilots to control all conventional aircraft. The single pilot mode was used for each of the UAS in the sector. The ATCo, conventional pseudopilots, and UAS pseudopilots spoke to each other using push-to-talk headsets over the voice server. The voice system was modified for the UAS stations. A fixed delay of $1.5 \mathrm{~s}$ was inserted before transmitting the UAS pseudopilots' message to the ATCo and conventional pseudopilots.

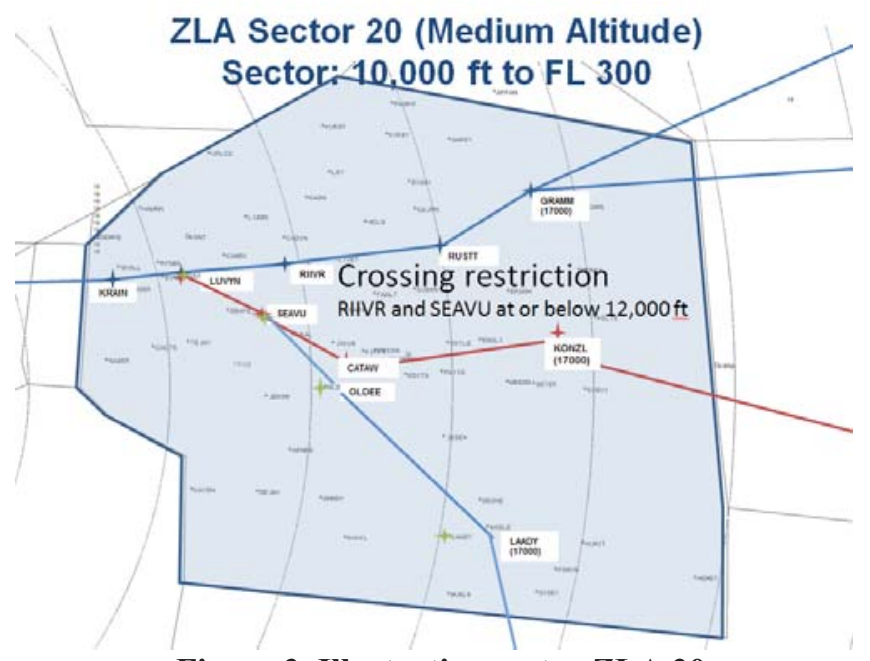

Figure 3. Illustration sector ZLA 20

Two parallel worlds were run at a time. Nine to 12 computers were required for each simulated world. The controller's station included two computers, one to simulate their radar scope and a second small display acting as 'Mission Control' to provide instructions to the controller regarding altitude changes to the UAS. Two stations were used for each conventional aircraft pseudopilot: one station was a "ghost" controller station that allowed them to check the traffic coming into the sector and the second to control all aircraft, with the exception of the UAS, within sector ZLA 20. One to four stations were used to control the UAS aircraft, depending on the number of UAS in the sector during each scenario. Additional computers were used to manage the simulation software, record communication data between the controller and pseudopilots over the voice server, and for the ADRS simulation hub. The voice software was also modified to simulate stepped-on transmissions so that when simultaneous transmissions occurred, the transmission would be unintelligible for everyone listening on that frequency.

\section{Procedure}

The simulation was conducted over two days for each participant. On the morning of the first day, participants completed consent forms and demographic questionnaires, and were briefed on simulation procedures. Following the briefing, the controllers worked three practice trials and nine experimental trials for the remaining time.

Each experimental trial lasted 40 minutes. During the trial, controllers managed all air traffic coming into their sector. Controllers were instructed that arrival traffic on approach to LAX had priority and were required to leave the sector at an altitude no greater than 11,000 feet, and at an air speed of 250 knots. The controllers were told that their sector had a Letter of Agreement to accommodate requests regarding the UAS in order to fulfill the UAS flight objectives while maintaining safe operations for all air traffic. All UAS were given a Predator callsign (e.g., PD-1) for the "slow" UAS and a Global Hawk callsign (e.g., GH-1) for the "fast" UAS.

Beginning approximately one to two minutes into the trial, and occurring every four to five minutes afterwards, either "Mission Control" or the UAS pseudopilot initiated a flight-change request to sector ATCo. Requests from "Mission Control," the screen to the right of the controller's radar scope, alerted the controller through his headset and display of a new, optimal altitude that the UAS should be flying to accomplish their mission objective (ex. "PD-1 climb and maintain 14000"). Mission control only requested altitude changes for the UAS. During the next period the UAS pseudopilot requested a speed change. A total of 8 requests for all UASs was completed each trial, four initiated by the controller and four by the UAS pseudopilot. When multiple UAS were in the sector, the total number of communications was divided evenly between each UAS.

Voice delays were inserted at the UAS pseudopilot station. Voice software at this station automatically held the UAS pilot's audio transmission for 1.5 seconds before broadcasting to the ATCo and conventional pseudopilots. 
The UAS station only included a transmission delay, not a receiving delay. The controller and conventional pseudopilots had no receiving or transmission delays.

After each trial, controllers rated their situation awareness [11], workload [12], and experience interacting with the conventional and UAS pseudopilots. For workload, the Once all nine experimental trials were completed, controllers answered post-simulation and debriefing questionnaires. Finally, the controllers were interviewed during a session covering the same topics as the debriefing survey.

\section{RESULTS}

A series of 3 (Number of UAS: 1, 2, or 4) X 3 (Speed: slow, mixed, fast) repeated measures ANOVAs were performed on ATCo's performance, workload, and acceptability ratings of UAS and conventional pilot verbal response delays.

\section{ATCo Performance}

ATCo performance was examined by the number of losses of separation (LOS; a safety metric) and distance it took the conventional AC to travel through the sector (an efficiency metric). Overall, the number of LOS averaged 2.6 ( $S E=$ .28). Although the number of LOS was not significantly affected by either number of UAS or speed, we did observe a significant linear trend between number of UAS and number LOS, $F(1,7)=5.42 ; p=.05$ (see Figure 4 ). The linear component accounted for $44 \%$ of the variance in LOS. Moreover, about half the number of LOS involved a UAS.

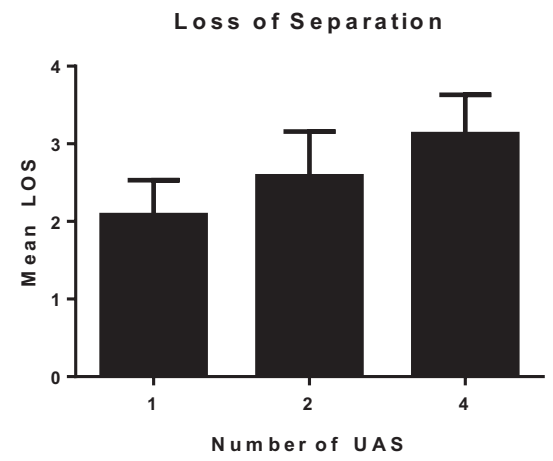

Figure 4. Mean Number of Losses of Separation as a function of Number of UAS

For distance traveled, there was a significant Number of UAS x Speed interaction, $F(4,28)=5.026, p=.004$, see Figure 5. For one UAS in the sector, the distance conventional AC travelled was not affected by UAS speed. However, when there were two UAS in the sector, the average distance conventional AC travelled through the sector was higher when the UAS was at slow compared to fast or mixed speeds. In contrast, when there were four UAS in the sector, the slow UAS decreased the average distance travelled relative to four mixed and four fast UAS.
In short, more efficient traffic flows occurred with faster speeds for two UAS in the sector, and with slower speeds when there were four UAS in the sector. This particular finding may be a result of the specific traffic pattern and UAS flight paths used in the present study.

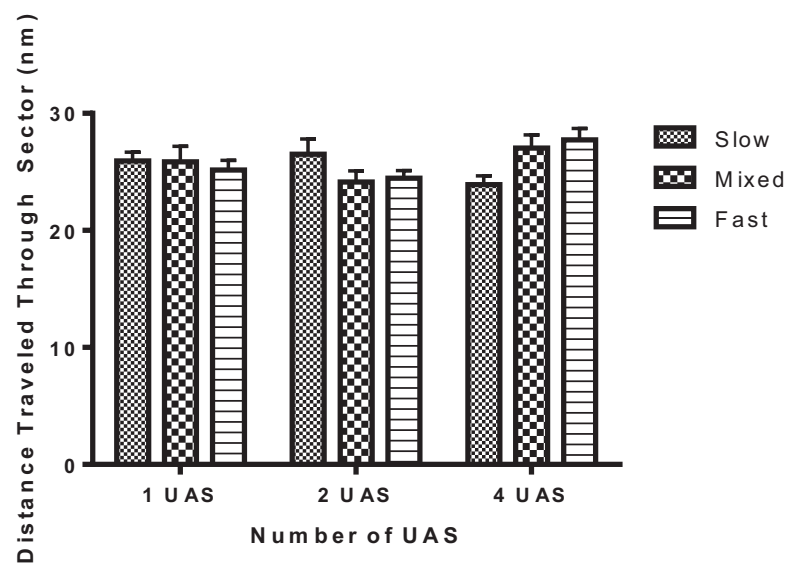

Figure 5. Number of UAS $x$ Speed interaction for distance AC traveled through the sector

\section{Workload}

Performance-based workload was examined through the hand-off accept times (shorter latencies correspond to lower workload), and subjective workload was examined with NASA-TLX ratings.

For handoff-accept time, there was a main effect of number of UAS, $F(2,14)=10.536, p=.002$, where the handoff accept time linearly decreased with the number of UAS, see Figure 6. The linear trend was again significant $F(1,7)=$ $16.1 ; p=.005$, and was likely caused by holding the average number of $\mathrm{AC}$ in the sector relatively constant at roughly eight $\mathrm{AC}$. Consequently, when four UAS, were in the sector, there were fewer conventional AC entering the sector.

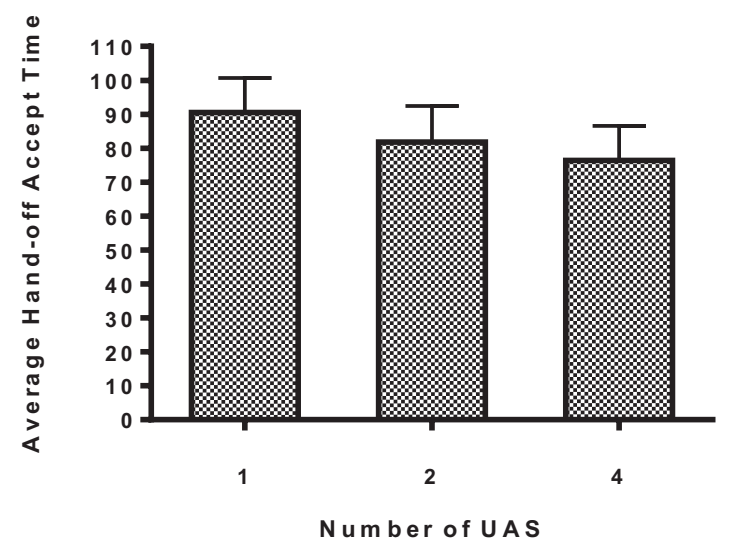

Figure 6. Main effect for Hand-off Accept Time x Number of UAS 


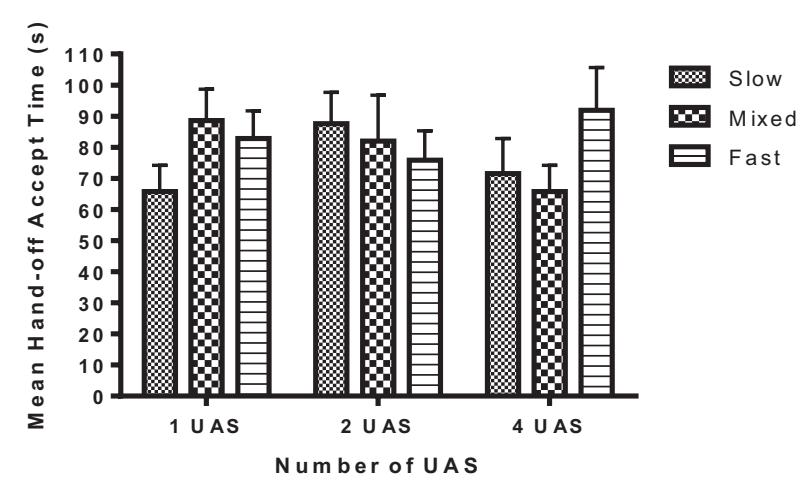

Figure 7. Number of UAS x UAS Speed interaction for Hand-off Accept Time

The main effect was modified by a significant Number of UAS $\times$ Speed interaction, $F(4,28)=3.16, p=.029$, see Figure 7. With a single UAS in the sector, the hand-off accept time was shorter when the UAS was slow. With two fast UAS, hand-off accept times were shorter than with two UAS traveling at slow or mixed speeds. However, with four UAS, constant slow or mixed speeds led to shorter hand-off accept times than constant fast speeds. Thus, workload was lower for slow UAS when there was one or four in the sector, but workload was higher for the slow UAS when there were two in the sector.

For the NASA-TLX, ATCos rated the overall workload as average $(\mathrm{M}=53)$. Only the main effect of Number of UAS, $F(2,14)=2.730, p=.025$ was significant. In contrast to the hand-off accept times, subjective workload was rated the lowest when there were two UAS in the sector $(M=49)$ compared with one $(M=56)$ or four $(M=55)$ UAS. This likely reflects key contextual factors of the scenarios. When there was only one UAS in the sector, there were many more aircraft entering the sector than in the other conditions, thereby increasing the perceived workload. On the other hand, when there were four UAS, although there were fewer aircraft entering the sector than when there were two UAS, there was more LOS, as shown above. Increasing the number of operational errors in the four UAS condition therefore could have affected the subjective assessment of the workload created by this condition, as ATCos more likely had to work harder to maintain safe separation minima.

\section{Acceptability Ratings}

ATCo acceptability ratings of pilot verbal delays were analyzed using a 3 (Number of UAS: 1, 2, or 4) X 3 (Speed: slow, mixed, fast) X 2 (Pilot: UAS, conventional) repeated measures ANOVA. Overall, all conditions were rated acceptable (above 4.0 on a scale of $1=$ not acceptable to $7=$ very acceptable). The ratings did not differ significantly as a function of number or speed of the UAS, or as a function of pilot type (see Table 1).
Table 1. ATCo Acceptability Ratings $(1=$ not acceptable to $7=$ very acceptable) for UAS and Conventional Pilot Verbal Response Latencies

\begin{tabular}{|c|c|c|}
\hline $\begin{array}{c}\text { Condition } \\
\text { (No. UAS-Speed) }\end{array}$ & UAS Pilots & Conventional Pilots \\
\hline 1-Slow & 5.25 & 5.38 \\
\hline 1-Mixed & 4.88 & 5.25 \\
\hline 1 -Fast & 4.88 & 6.25 \\
\hline 2-Slow & 5.50 & 5.63 \\
\hline 2-Mixed & 5.13 & 5.63 \\
\hline 2-Fast & 5.25 & 6.38 \\
\hline 4-Slow & 5.13 & 5.75 \\
\hline 4-Mixed & 4.75 & 6.25 \\
\hline 4-Fast & 5.13 & 5.75 \\
\hline
\end{tabular}

\section{DISCUSSION}

The present study inserted an additional $1.5 \mathrm{~s}$ verbal response delay into UAS pilot communications, a value that previously resulted in an acceptable rating of verbal response latencies [8]. However, we increased the number and speeds of UAS flying in the sector to determine whether the safety and efficiency with which ATCos managed traffic would be affected. We also examined ATCO's acceptability ratings of the delays under these conditions.

We found negative effects of the number of UAS on a measure of safety, the number of LOS. Although there were no significant differences in LOS, the linear trend observed indicates that safety is compromised with increasing numbers of LOS in the sector. Moreover, we found that a UAS was involved in at least half of the LOS occurrences, again suggesting that the increasing number of UAS did negatively impact the ATCos' performance to some degree. In addition, the efficiency with which the ATCos managed traffic was affected by the number and speeds of the UAS. Specifically, we found an interaction between number of UAS and speed, such that whether there were two or four UAS determined if faster UAS speed was related to greater distance of other aircraft through the sector or lesser distance through the sector. This shows that the effect of a single variable like speed depends on other contextual factors.

With respect to workload, we found that the more UAS in the sector, the lower the hand off accept time, an objective measure of workload. Prima facie this seems counterintuitive. However, we need to keep in mind that as the average number of aircraft (including UAS) in the sector was held relatively constant. That is, when there were more UAS present, there were fewer conventional aircraft entering the sector, and thus fewer hand-off- 
acceptances were required. This reduction in the number of aircraft being handed off may explain the reduction in hand-off accept time. Additionally, ATCos reported that managing the conventional aircraft required more interactions than managing the UAS because the latter, even when fast moving, were significantly slower than the conventional aircraft. Thus, ATCos reported generally moving all conventional air traffic around the UAS, avoiding as much as possible, interactions with the UAS. Researchers should be mindful of contextual factors, ATCo strategies, and other performance tradeoffs when evaluating the impacts of UAS in the NAS.

Finally, we found that the ratings of acceptability of the UAS pilot verbal latencies were not affected by the number or speeds of the UAS. This suggests that delays in verbal responding that are $1.5 \mathrm{sec}$ longer than normal are still acceptable to controllers. Interestingly, ATCos gave equivalent ratings to both UAS pilots and conventional aircraft pilots, despite the fact that the delay of $1.5 \mathrm{sec}$ was only inserted in the former. This finding is similar to the results of $\mathrm{Vu}$ et al. [8] and shows that when controllers judge the acceptability of delays, they likely take into account other sector (e.g., traffic density) and performance (e.g., LOS) factors when rating acceptability of the measured response.

\section{ACKNOWLEDGMENTS}

This project was supported by NASA cooperative agreement NNX12AH23A, NASA UAS in the NAS (Jay Shively and Walter Johnson, Technical Monitors), and NASA cooperative agreement NNX09AU66A, "Group 5 University Research Center: Center for Human Factors in Advanced Aeronautics Technologies" (Brenda Collins, technical monitor).

\section{REFERENCES}

1. Merlin, P. W. (2009). Ikhana unmanned aircraft system Western states fire missions. Washington, DC: National Aeronautics and Space Administration.

2. ICAO. (2011). Unmanned Aircraft Systems (UAS) (ICAO Cir 328). Montreal, Canada: International Civil Aviation Organization.

3. Shively, R. J., Vu, K.-P. L., \& Buker, T. J. (2013). Unmanned Aircraft System Response to Air Traffic Control Clearances: Measured Response. Proceedings of the Human Factors and Ergonomics Society Annual Meeting, 57(1), 31-35. doi:10.1177/1541931213571009
4. Williams, K. (2007). An assessment of pilot control interfaces for unmanned aircraft. (Report no. DOT/FAA/AM-07/8). Washington, DC: U.S. Department of Transportation, Federal Aviation Administration, Office of Aerospace Medicine.

5. Merlin, P. W. (2013). Crash course: Lessons learned from accidents involving remotely piloted and autonomous aircraft. Washington, DC: National Aeronautics and Space Administration. HCI-Aero Conference Publications Format. Available at http://www.ihmc.us/hci-aero2010/hciaero2010/Formats.html

6. Rantanen, Esa M., McCarley, J. S., \& Xu, X. (2004). Time Delays in Air Traffic Control Communication Loop: Effect on Controller Performance and Workload. The International Journal of Aviation Psychology, 14(4), 369-394. doi:10.1207/s15327108ijap1404_3

7. Prinzo, O., Hendrix, A., \& Hendrix, R. (2009). The outcome of ATC message length and complexity on en route pilot readback performance, (January). Retrieved from

http://oai.dtic.mil/oai/oai?verb=getRecord\&metadataPrefi $\mathrm{X}=$ html\&identifier $=$ ADA494551

8. Vu, K.-P. L., Chiappe, D., Morales, G., Strybel, T. Z., Battiste, V., Shively, R. J., \& Buker, T. J. (under review). Impact of UAS Pilot Communication and Execution Latencies on Air Traffic Controller's Acceptance of UAS Operations. Manuscript submitted for publication.

9. Fern, L., \& Shively, R. J. (2009). A comparison of varying levels of automation on the supervisory control of multiple UASs. In Proceedings of AUVSI's Unmanned Systems North America 2009, Washington, D.C.

10. Prevot, T. (2002). Exploring the many perspectives of distributed air traffic management: The Multi Aircraft Control System: MACS. International Conference on Human-Computer Interaction in Aeronautics, HCI-Aero 2002, 23-25.

11.Taylor, R. M. (1990). Situational Awareness Rating Technique (SART): The development for a tool for aircrew systems design (AGARD-CP-478). In Situation awareness in aerospace operations (pp. 3/1-3/17). Neuilly Sur Seine, France: NATO-AGARD.

12.Hart, S. G., \& Staveland, L. E. (1987). Development of NASA-TLX (Task Load Index): Results of empirical and theoretical research. In P. A. Hancock \& N. Meshkati (Eds.), Human mental workload (pp. 139-183). Amsterdam, The Netherlands: Elsevier. 